\title{
Política Nacional de Saúde Bucal Brasileira (Brasil Sorridente): Um resgate da história, aprendizados e futuro*
}

Brazilian National Oral Health Policy (Smiling Brazil): A rescue of history, learning and future

Política Nacional de Salud Bucal Brasileña (Brasil Sonriente): Un rescate de la historia, el aprendizaje y el futuro para ser compartidos

Fecha de recepción: 30-08-2018 | Fecha de aceptación: 18-02-2019

\begin{abstract}
Maristela Honório Cayetano
Faculdade de Odontologia da Universidade de São Paulo, São Paulo, Brasil. maricayetano@usp.br. https://orcid.org/0000-0002-0694-4171
\end{abstract}

\author{
Fernanda Campos de Almeida Carrer \\ Faculdade de Odontologia da Universidade de São Paulo, São Paulo, Brasil. fernandacsa@usp.br. \\ https://orcid.org/0000-0003-3745-2759
}

\section{Mariana Gabriel}

Faculdade de Odontologia da Universidade de Mogi das Cruzes, Mogi das Cruzes, Brasil. marianagabrielodonto@gmail.com. https://orcid.org/0000-0001-8824-5827 


\section{FABio CARneiro MARTins}

Faculdade de Odontologia da Universidade de São Paulo, São Paulo, Brasil. fabio.carneiro.martins@usp.br. https://orcid.org/0000-0002-7014-9439

\section{Gilberto Alfredo Pucca Jr.}

Universidade de Brasília, Brasília, Brasil. gilbertopucca@unb.br. https://orcid.org/0000-0002$\underline{8781-9857}$

\section{Maria Ercilia de Araujo}

Faculdade de Odontologia da Universidade de São Paulo, São Paulo, Brasil. mercilia@usp.br. https://orcid.org/0000-0003-2689-2556

*Investigación original

Correspondencia: maricayetano@usp.br; fernandacsa@usp.br;

marianagabrielodonto@gmail.com; fabio.carneiro.martins@ usp.br; gilbertopucca@unb.br; mercilia@usp.br

doi: $\underline{\text { https://doi.org/10.11144/Javeriana.uo38-80.pnsb }}$

Cómo citar: Cayetano MH, Carrer FC, Gabriel M, Martins FC, Pucca Jr. GA. Política Nacional de Saúde Bucal Brasileira (Brasil Sorridente): Um resgate da história, aprendizados e futuro. Univ Odontol. 2019 ene-jun; 38(80). https://doi.org/10.11144/Javeriana.uo38-80.pnsb 


\section{RESUMO}

Antecedentes: no Sistema Único de Saúde do Brasil (SUS) a atenção básica é ordenadora do cuidado em saúde e representa o eixo estruturante do sistema. Em 2004, quando o Brasil Sorridente (BS) foi criado, o governo federal assumiu um importante papel de indução do crescimento da oferta de serviços de saúde bucal nos municípios e estados, criando linhas de financiamento específicos para criação de novas equipes de saúde bucal, para construção e implantação de centros de atenção secundária (Centros de Especialidades Odontológicas - CEO) e terciária dentre outras ações, que fizeram do BS uma rede de atenção à saúde e uma das maiores políticas públicas de saúde bucal do mundo. Objetivo: este artigo tem como objetivo compartilhar parte dessa experiência, a fim de promover reflexão em torno da inserção da saúde bucal nos sistemas de saúde ao redor do mundo, com especial interesse nos países da América Latina. Métodos: este é um estudo de caso do Brasil e as informações apresentadas nesse estudo foram coletas por meio de relatórios governamentais, base de dados secundários, artigos publicados e informantes chave. Resultados e conclusão: a experiência do Brasil vem sendo motivadora e mostrou que, além de necessário é possível inserir a saúde bucal nos sistemas de saúde universais. Porém, após 14 anos e com a instabilidade política atual do país, o futuro do Brasil Sorridente dependerá do compromisso dos formuladores de políticas, a continuidade do engajamento dos diferentes atores envolvidos, objetivos claros e estratégias transparentes e fundamentadas em evidências científicas para alcançá-los.

\section{Palavras-chave}


assistência à saúde; Brasil; epidemiologia nos serviços de saúde; odontologia comunitária; política pública; saúde bucal; sistemas de saúde

\section{Áreas temáticas}

odontologia; políticas públicas de saúde bucal; saúde pública

\section{ABSTRACT}

Background: in the Brazilian Unified Health System (SUS), primary health care coordinates and structures the public health care. In 2004, when the Smiling Brazil (SB) was created, the federal government assumed an important role of inducing the growth of the supply of oral health services in the municipalities and states, creating specific funding lines for the creation of new oral health teams, for the construction and implantation of secondary care centers (Dental Specialties Centers - CEO) and tertiary care (in hospitals), among other actions, that made SB a health care network and one of the largest public health policies in the world. Purpose: this study aims to share part of this experience, in order to promote reflection on the insertion of oral health in public health systems around the world, with special interest in the countries of Latin America. Methods: this is a Brazil case study, the information presented in this study was collected through government reports and databases, published articles and key informants. Results and Conclusion: The experience of Brazil has been motivating and showed that, it is possible to insert oral health in universal public health systems. However, after 14 years and with the current political instability of the country, the future of SB will depend on the commitment of the policymakers, the 
engagement of the different actors involved, clear objectives and transparent strategies based on scientific evidence.

\section{Keywords}

Brazil; community dentistry; community health services; delivery of health care; health services research; health systems; oral health; public policy

\section{Thematic fields}

dentistry; oral health public polices; public health

\section{RESUMEN}

Antecedentes: en el Sistema Único de Salud del Brasil, la atención básica es ordenadora del cuidado en salud y representa el eje estructurante del sistema. En 2004, cuando se creó Brasil Sonriente, el gobierno federal asumió el papel de inducción del crecimiento de la oferta de servicios de salud bucal en municipios y estados, creando líneas de financiamiento específicas para crear nuevos equipos de salud bucal, construir e implantar centros de atención secundaria (centros de especialidades odontológicas) y terciaria entre otras acciones, que hicieron del programa una red de atención a la salud y una de las mayores políticas públicas de salud bucal del mundo. Objetivo: compartir parte de esa experiencia, a fin de promover reflexión en torno a la inserción de la salud bucodental en los sistemas de salud alrededor del mundo, con especial interés en los países de América Latina. Métodos: este es un estudio de caso de Brasil y las informaciones presentadas en ese estudio fueron recolectadas a través de informes gubernamentales, base de datos 
secundarios, artículos publicados e informantes clave. Resultados y conclusión: la experiencia de Brasil viene siendo motivadora y mostró que, además de necesario es posible insertar la salud bucal en los sistemas de salud universales. Sin embargo, después de 14 años y con la inestabilidad política actual del país, el futuro de Brasil Sonriente dependerá del compromiso de los formuladores de políticas, la continuidad del compromiso de los diferentes actores involucrados, objetivos claros y estrategias transparentes y fundamentadas en evidencias científicas para alcanzarlos.

\section{Palabras clave}

asistencia a la salud; Brasil; epidemiología en los servicios de salud; odontología comunitaria; política pública; salud bucal; sistemas de salud

\section{Áreas temáticas}

odontología; políticas públicas de salud bucal; salud pública

\section{INTRODUÇÃO}

Nas últimas décadas, o Brasil evoluiu de um sistema de saúde excludente e mutilador, no qual apenas aqueles que contribuíam para o sistema de seguridade social tinham direito à assistência de

saúde, para um sistema universal, chamado Sistema Único de Saúde (SUS), no qual a saúde é um direito de todos e um dever do Estado, garantido na Constituição de 1988 (1). 
No SUS, a atenção básica é ordenadora do cuidado em saúde e representa o eixo estruturante do sistema, de acordo com as diretrizes da OPAS/OMS (2). O que se busca é a garantia da integralidade do cuidado e da universalização do acesso, por meio da Estratégia de Saúde da Família (ESF). Apesar dos avanços que ocorreram desde 1988, quando o SUS foi criado, é evidente que este é um sistema que enfrenta enormes desafios, entre eles, a grande diversidade socioeconômica da população, em um país com dimensões continentais e o subfinaciamento do sistema de saúde desde sua criação (3).

Outro avanço que merece destaque é a inserção da saúde bucal no SUS, por meio de uma política específica e articulada chamada "Brasil Sorridente" (Política Nacional de Saúde Bucal) (4). Uma das principais características dessa política é a ênfase em ações intersetorais e interministerias. Em 2004, ano em que o Brasil Sorridente (BS) foi criado, o governo federal assumiu um importante papel de indução do crescimento da oferta de serviços de saúde bucal nos municípios e estados, criando linhas de financiamento específicas para a criação de novas Equipes de Saúde Bucal - eSB (cirurgião dentista + técnico de saúde bucal e/ou auxiliar de saúde bucal), para construção e implementação de centros de atenção secundária e terciaria, dentre outras ações, que fizeram do Brasil Sorridente uma das maiores políticas públicas de saúde bucal do mundo (5).

Na América Latina muitos países possuem políticas públicas de saúde bucal, tais como Chile, Colômbia, Paraguai, Uruguai, portanto, as experiências acumuladas dessas nações, que possuem características socioeconômicas e geopolíticas semelhantes, podem contribuir para o fortalecimento dessas iniciativas com consequente melhoria das condições de saúde da população Latino-Americana (6). Tendo em vista o histórico de uma das mais ousadas políticas públicas de 
saúde bucal, este artigo tem como objetivo compartilhar parte dessa experiência, através da descrição do caso da política pública de saúde bucal Brasil Sorridente, a fim de promover reflexão em torno da inserção da saúde bucal nos sistemas de saúde ao redor do mundo, com especial interesse nos países da América Latina. As informações apresentadas nesse estudo foram coletadas por meio de relatórios governamentais, bases de dados secundários, artigos publicados e informantes chave.

\section{DESCRIÇÃO E ANÁLISE DO CASO BRASIL SORRIDENTE}

\section{Como a rede de Atenção à Saúde Bucal está organizada no Brasil?}

No que se refere ao modelo em que a Odontologia está inserida no sistema público de saúde atualmente, é importante destacar que o Brasil Sorridente está inserido no Sistema de Redes de Atenção à Saúde (RAS), como uma rede poliárquica que visa a integralidade do cuidado, equidade e a articulação (entre todos os níveis de atenção e com outras redes temáticas) (7) (Figura 1).

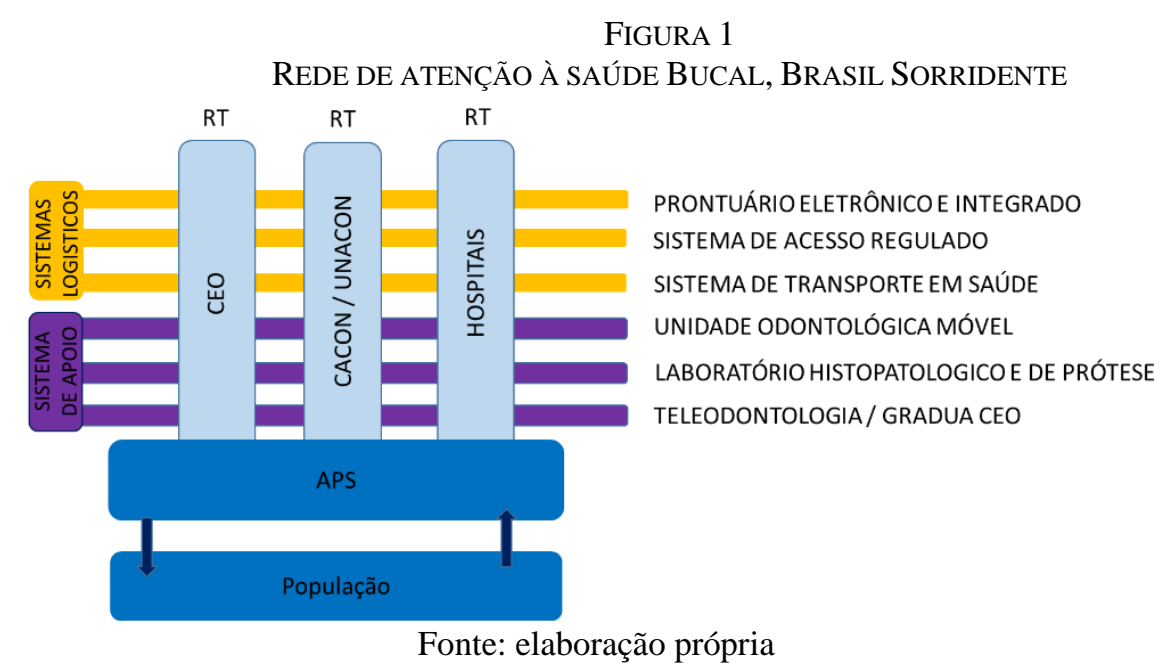

A saúde bucal se inseriu nessa rede de maneira bastante eficiente e os maiores avanços foram no sentido da institucionalização da política e na infraestrutura da capacidade instalada dos serviços 
de saúde bucal, nos três níveis de atenção (atenção básica, média complexidade e alta complexidade) (8).

Depois de institucionalizar a Política Brasileira de Saúde Bucal "Brasil Sorridente”, com leis, portarias e normas específicas, foi necessário substituir equipamentos sucateados e ampliar a estrutura física da rede de saúde bucal em construção para receber esse novo serviço, aprimorando sua capacidade instalada. Outra importante vertente se deu na formação dos Recursos Humanos em Saúde, uma vez que a política induziu mudanças estruturais no processo de trabalho, por meio de uma atuação multiprofissional, com foco na prevenção e em uma atuação voltada para o serviço público, diferente da lógica da clínica curativa e individual da profissão caracterizada até o momento $(9,10)$.

Tendo em vista o compromisso da política com a integralidade do cuidado e com a Atenção Primária à Saúde (APS), ampliou-se as eSB (Cirurgião-dentista + Técnico de Saúde Bucal +e/ou Auxiliar de Saúde Bucal), de modo que estivessem articuladas às equipes de saúde da família (Médico, Enfermeiro, Auxiliar de Enfermagem, Agente Comunitário de Saúde) e distribuídas por todo o território nacional. Antes da Política, em 2003, eram 6,170 equipes de saúde bucal em 2,787 municípios, e em 2017 as equipes se somavam em 24,053, espalhadas em 4,933 municípios dos 5561 existentes no país, o que impactou diretamente na cobertura populacional da saúde bucal $(11,12)$. 
O Brasil possui regiões de difícil acesso, muitas vezes com populações ribeirinhas isoladas que necessitam de especial atenção, para tanto, foram criadas as Unidades Odontológicas Moveis (UOM) (5).

Além de aspectos relativos à assistência, o BS tem um importante componente de prevenção e promoção à saúde junto ao Programa de Saúde na Escola (PSE), que é uma ação interministerial entre a educação e a saúde, com foco em ações de vigilância em saúde, a partir de uma equipe multidisciplinar no ambiente escolar e assistência ofertada nas Unidades de Saúde e nas escolas (13). Em 2017, nove anos após sua implementação, 18 milhões de escolares foram atendidos em ações de saúde em todo o Brasil (14). Na saúde bucal, essa iniciativa inclui diversas estratégias, entre elas, o diagnóstico situacional dos escolares por meio da classificação de risco (Tabela 1), educação em saúde, escovação supervisionada (na maioria das vezes disponibilizando aos alunos escova dental e dentifrícios), aplicação tópica de flúor (quando indicado), tratamento restaurador atraumático (ART), entre outras ações identificadas no território de atuação. O PSE, é apenas um exemplo, pois o Brasil Sorridente se apresenta de forma transversal em outras ações do Ministério da Saúde.

TABELA 1

CLASSES DE RISCO DE CÁRIE DENTÁRIA E CRITÉRIOS PARA INCLUSÃO SEGUNDO A SITUAÇÃO INDIVIDUAL

\begin{tabular}{|c|c|c|}
\hline Classificação & Grupo & $\begin{array}{l}\text { Situação individual } \\
\end{array}$ \\
\hline Baixo risco & $\mathrm{A}$ & $\begin{array}{l}\text { Ausência de lesão de cárie, sem placa, sem gengivite e/ou sem } \\
\text { mancha branca ativa }\end{array}$ \\
\hline \multirow[t]{2}{*}{$\begin{array}{l}\text { Risco } \\
\text { moderado }\end{array}$} & B & $\begin{array}{l}\text { História de dente restaurado, sem placa, sem gengivite e/ou sem } \\
\text { mancha branca ativa }\end{array}$ \\
\hline & $\mathrm{C}$ & $\begin{array}{l}\text { Uma ou mais cavidades em situação de lesão de cárie crônica, } \\
\text { mas sem placa, sem gengivite e/ou sem mancha branca ativa }\end{array}$ \\
\hline \multirow[t]{3}{*}{ Alto risco } & D & $\begin{array}{l}\text { Ausência de lesão de cárie ou presença de dente restaurado, mas } \\
\text { com presença de placa, de gengivite e/ou de mancha branca ativa }\end{array}$ \\
\hline & $\mathrm{E}$ & Uma ou mais cavidades em situação de lesão de cárie aguda \\
\hline & $\mathrm{F}$ & Presença de dor e/ou abscesso \\
\hline
\end{tabular}

Fonte: Secretaria Municipal da Saúde de São Paulo (15) 
Para garantir a linha de cuidado do usuário, além da reestruturação da atenção primária, o Brasil Sorridente criou os Centros de Especialidades Odontológicas (CEO), que oferecem atendimento nas especialidades de cirurgia oral menor, diagnóstico de câncer de boca e outras doenças de tecidos moles, periodontia, atenção aos pacientes com necessidades especiais e endodontia (Figura 2). Em 2010 foram acrescidos aos CEO tratamentos de ortodontia e implantes dentários (16). Além disso, iniciou-se a implantação dos Laboratórios Regionais de Prótese Dentária (LRPD), a fim de responder à demanda da população adulta e idosa do Brasil e garantir a integralidade do cuidado.

FIGURA 2

NÚMERO DE CEO IMPLEMENTADOS NO BRASIL

Entre 1 e 2

Mais de 2

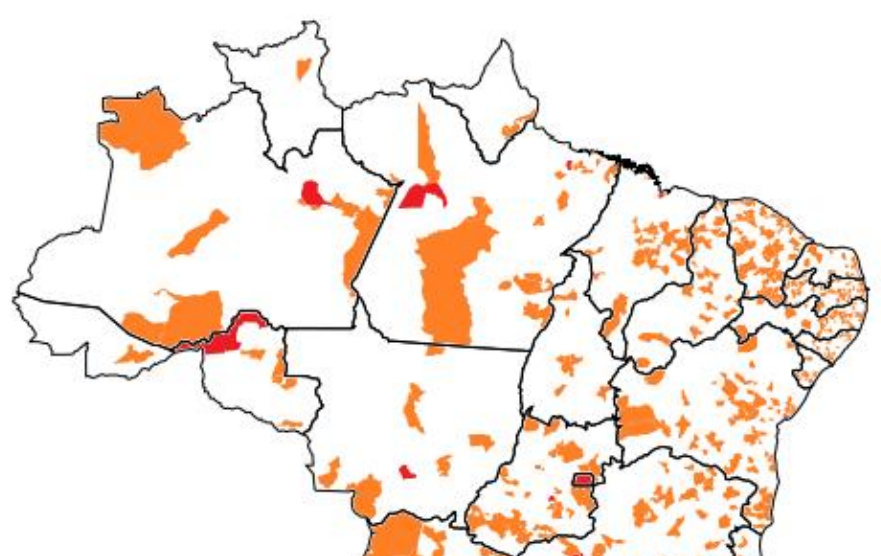

Fonte: Sala de Apoio à Gestão Estratégica - SAGE (17)

Em janeiro de 2018, o Ministério da Saúde registrou 1069 CEO, que obrigatoriamente, contam com serviços de diagnóstico bucal, com ênfase para o câncer de boca (17). Para dar suporte a esses centros, a rede foi estruturada com serviços de análise de exames histopatológicos, para emissão 
de laudos anatomopatológicos. Destaca-se que os CEO, além de unidades assistenciais de média complexidade, são estruturadores da atenção primária, pois são ambulatórios de referência e contra referência, tanto para ela como para a atenção terciária.

Para compor todos os componentes da rede, o BS investiu na organização da alta complexidade, ou seja, cuidado hospitalar a pacientes com tumores benignos bucomaxilofaciais, pacientes especiais que necessitam de cuidado sob anestesia geral, cuidados odontológicos à pacientes submetidos à quimioterapia e radioterapia, prótese bucomaxilofacial, pacientes crônicos e hospitalizados para procedimentos cirúrgicos.

O Brasil é um país com alta prevalência de câncer de boca (18) e para atender o paciente oncológico, incluindo os portadores de câncer de cabeça e pescoço, foram criados as Unidades de Assistência de Alta Complexidade em Oncologia (UNACON) e de Centros de assistência especializada em oncologia (CACON) (19), que recebem os pacientes com diagnóstico confirmado de câncer de boca dos Centros de Especialidades Odontológicas, especialmente dos serviços de estomatologia. O SUS também oferece serviços de reabilitação ao paciente com mutilação de cabeça e pescoço.

Mais recentemente, em 2016, o Brasil Sorridente lançou o programa Gradua-CEO, que articula os serviços de saúde bucal oferecidos pelas universidades à rede de saúde bucal do Sistema Único de Saúde, pois desse modo a Universidade integra a rede e o estudante vivencia o serviço público durante sua formação. 
Para a implementação da Política de Saúde Bucal, incrementou-se o financiamento. Em 2002 foram destinados cerca de US\$ 16 milhões para área de saúde bucal, já em 2016 este valor foi de US\$ 206.938.620 milhões.

O surgimento do BS impactou também o mercado de trabalho, pois aumentou em $50 \%$ o número de Cirurgiões-Dentistas no SUS, fazendo com que atualmente, cerca $50 \%$ dos dentistas inscritos no Cadastro Nacional de Estabelecimentos de Atenção à Saúde - CNES tenha vínculo com o serviço público, ou seja, 58.240 cirurgiões-dentistas (12).

Um dado muito importante que justificou a necessidade de se instituir uma política de saúde bucal no Brasil foi revelado em 2003 pela Pesquisa Nacional de Amostra por Domicílios (PNAD), que revelou que 27,9 milhões (16 \%) dos brasileiros nunca tinham sido consultados por um Cirurgião Dentista (20). Além da falta de acesso de parte da população, o BS se deparou na sua criação com a precariedade da saúde bucal da população brasileira, demonstrada pelos levantamentos epidemiológicos de saúde bucal que aconteceram nos anos de 1986, 1996, 2003 (21).

Em 2010, como uma ação de vigilância em saúde bucal integrante do Brasil Sorridente, o Ministério da Saúde realizou o SB-2010, levantamento epidemiológico nacional, seguindo a padronização da Organização Mundial de Saúde (OMS) para aferir os primeiros impactos da Política Nacional de Saúde Bucal após sete anos de implantação e pode-se observar mudança positiva no perfil epidemiológico $(22,23)$. Dentre os principais resultados desse levantamento, observa-se que o país passou a fazer parte do grupo de países com baixa prevalência de cárie aos 12 anos, segundo critérios da Organização Mundial de Saúde (OMS), ao reduzir o índice de dentes 
cariados, perdidos ou obturados (CPO-D) de 2,8 em 2003 para 2,1 em 2010. Além disso, o número de adolescentes e adultos que sofreram algum tipo de perda dentária foi reduzido em $50 \%$.

Após 10 anos da política, mais de 7 milhões de pessoas passaram a ter acesso a água tratada e fluoretada e $44 \%$ das crianças aos 12 anos estavam livres de cárie (5). A tabela 2 demonstra a evolução dos dados epidemiológicos nas idades índices. Em 2010, na faixa etária de 35 a 44 anos, o componente cariado decresceu de 2,33 para 1.48, enquanto que o componente obturado evoluiu de 4,22 para 7.33, demonstrando maior acesso ao tratamento clinicamente conservador (Tabela 2). Entretanto, embora os indicadores tenham demonstrado avanços na política de saúde bucal, eles também trouxeram um alerta em relação aos desafios para tratar as sequelas de um modelo de cuidados equivocado, que mutilou adultos e idosos no passado. Para dar resposta a essas necessidades, o Brasil Sorridente precisou desenvolver ações estratégicas, que resultaram na construção de uma rede de atenção à saúde bucal, integrada e articulada com o Sistema Único de Saúde brasileiro que descreveremos a seguir.

TABELA 2

SITUAÇÃO DO CPO-D DA POPULAÇÃO BRASILEIRA, SEGUNDO AS IDADES ÍNDICES NOS ANOS DE 2013 E 2010

\begin{tabular}{llll}
\hline Idade Índice & Situação & $\mathbf{2 0 0 3}$ & $\mathbf{2 0 1 0}$ \\
\hline \multirow{5}{*}{$\mathbf{5}$ anos } & $\mathbf{H}$ & 16,05 & 16,3 \\
& $\mathbf{C}$ & 2,3 & 1,95 \\
& $\mathbf{O} / \mathbf{C}$ & 0,06 & 0,08 \\
& $\mathbf{O}$ & 0,36 & 0,33 \\
& $\mathbf{P}$ & 0,08 & 0,06 \\
& $\mathbf{C E O}-\mathbf{D}$ & 2,80 & 2,43 \\
\hline \multirow{3}{*}{$\mathbf{1 2}$ anos } & CEO $=\mathbf{0}$ & $40,6 \%$ & $46,6 \%$ \\
& $\mathbf{H}$ & 22,7 & 23,18 \\
& $\mathbf{C}$ & 1,62 & 1,12 \\
\hline & $\mathbf{O} / \mathbf{C}$ & 0,07 & 0,09 \\
\hline
\end{tabular}




\begin{tabular}{|c|c|c|c|}
\hline & $\mathbf{P}$ & 0,18 & 0,12 \\
\hline & CPO-D & 2,78 & 2,07 \\
\hline & $\mathrm{CPO}=0$ & $31,08 \%$ & $43,5 \%$ \\
\hline \multirow{7}{*}{ 15-19 anos } & $\mathbf{H}$ & 22,24 & 24,09 \\
\hline & C & 2,60 & 1,52 \\
\hline & $\mathrm{O} / \mathrm{C}$ & 0,19 & 0,18 \\
\hline & $\mathbf{O}$ & 2,49 & 2,16 \\
\hline & $\mathbf{P}$ & 0,89 & 0,38 \\
\hline & CPO-D & 6,17 & 4,25 \\
\hline & $\mathrm{CPO}=0$ & $11,06 \%$ & $23,9 \%$ \\
\hline \multirow{7}{*}{ 35-44 anos } & $\mathbf{H}$ & 10,85 & 13,53 \\
\hline & C & 2,33 & 1,48 \\
\hline & $\mathrm{O} / \mathrm{C}$ & 0,35 & 0,46 \\
\hline & $\mathbf{O}$ & 4,22 & 7,33 \\
\hline & $\mathbf{P}$ & 13,23 & 7,48 \\
\hline & CPO-D & 20,13 & 16,75 \\
\hline & $\mathrm{CPO}=0$ & $0,52 \%$ & $0,9 \%$ \\
\hline \multirow{7}{*}{$65-74$ anos } & $\mathbf{H}$ & 3,4 & 3,6 \\
\hline & $\mathbf{C}$ & 1,17 & 0,52 \\
\hline & $\mathrm{O} / \mathrm{C}$ & 0,06 & 0,10 \\
\hline & $\mathbf{O}$ & 0,73 & 1,62 \\
\hline & $\mathbf{P}$ & 25,83 & 25,29 \\
\hline & CPO-D & 27,79 & 27,53 \\
\hline & $\mathrm{CPO}=0$ & $0,52 \%$ & $0,2 \%$ \\
\hline
\end{tabular}

Fonte: Elaboração própria com dados disponíveis em SB Brasil 2010: Pesquisa Nacional de Saúde Bucal: resultados principais

Em 2015, o Brasil realizou o levantamento epidemiológico apenas no estado de São Paulo, o SB/São Paulo, onde 17.560 pessoas foram examinadas em 163 municípios. Nesse levantamento foram examinados 3 grupos etários (15-19, 35-44 e 65 anos ou mais). Na idade de 15 à 19 anos, o CPOD médio do estado foi de 3,57, sendo 1,25 componentes cariados, 0,21 obturado e cariado, 0,15 extraído e 1,96 obturados. De 35 a 44 anos obteve-se um CPOD médio de 15,84, sendo 1,53 componentes cariados, 0,54 obturado e cariado, 6,30 extraído e 7,46 obturados. Por fim, na idade de 65 anos ou mais o CPOD médio foi de 28,22, sendo 0,59 componentes cariados, 0,18 obturado 
e cariado, 25,87 extraído e 1,57 obturados (24). Há possibilidade de realização de outro SB Brasil, nacional em 2020.

\section{DISCUSSÃO E CONCLUSÕES}

\section{Considerações em relação as barreiras e facilitadores da implementação da rede de saúde bucal na perspectiva dos aprendizados e do futuro}

Considerando o período de 14 anos da política Brasil Sorridente, os achados demostram importantes avanços na saúde bucal brasileira, tanto em relação acesso aos serviços como no impacto epidemiológico. Certamente a implementação da política foi um marco na odontologia brasileira, tento em vista que antes as ações eram especificas para algumas faixas etárias, como por exemplo, para crianças e gestantes e voltados para os tratamentos curativos. Nos últimos anos o país apresentou uma conjuntura política favorável para a inserção da odontologia no SUS, ou seja, em um sistema de saúde pautado na universalidade, integralidade e equidade. Em 2003 a saúde bucal entrou na agenda governamental e a política foi formulada e implementada no país. Vale ressaltar, que embora a saúde bucal seja compreendida como fundamental para a saúde geral, poucos países ao redor do mundo incluem a odontologia em seus sistemas públicos de saúde, o que faz do caso brasileiro um modelo inovador de sistema integral e universal. Entretanto, vale ressaltar que este é um processo em construção e que a rede de atenção em saúde bucal precisa ser ampliada, aprimorada e sedimentada para que o Brasil Sorridente se transforme em uma política de Estado e não de governos, e que com isto, resista como política perene principalmente em épocas de crise econômica e política como a que estamos vivenciando atualmente (25). 
Em relação aos dados epidemiológicos, observa-se uma diminuição da carga de doenças bucais da população brasileira, entre eles a queda do componente cariado e o aumento do componente obturado no índice CPOD, aumento do número de crianças livres de cárie, uma população adulta com mais dentes na boca, entre outros. No entanto, essa melhora não se apresenta de maneira homogênea na população, sendo notórias as discrepâncias relacionadas a disponibilidades do serviço e as piores condições epidemiológicas em locais com maior vulnerabilidade social, o que certamente impõe importantes desafios futuros à política e maior interdisciplinaridade com as medidas sociais e econômicas do país (26). A política também está sendo fundamental para o monitoramento das condições epidemiológicas da saúde bucal da população brasileira, por meio dos levantamentos padronizados da OMS, pois essa é uma importante lacuna internacional e agravada na américa latina, que apresenta dados não padronizados e desatualizados.

A estruturação da rede está organizada em diferentes níveis de atenção, contemplando medidas de promoção, prevenção e tratamento em uma lógica de atuação multidisciplinar. Para alguns atores envolvidos na rede de saúde bucal, ela ainda se apresenta de forma inicial e deslocada das outras redes de atenção, mas o fortalecimento da atenção primária à saúde, com as eSB, e a continuidade da atenção por meio dos CEO, vem contribuindo para essa estruturação (27). Alguns componentes da rede, como por exemplo, o gradua CEO, apresentam grande potencial transformador do modelo de atenção à saúde bucal, pois, no exemplo citado, integra ensino e serviço público, de modo a institucionalizar as vivências dos estudantes de odontologia no SUS. No entanto, ainda apresenta uma implementação incipiente que precisa ser fortalecida. Por outro lado, ainda na área da educação em odontologia, a implementação das Diretrizes Curriculares Nacionais (DCN) apresentou grande contribuição para a política nacional de saúde bucal, uma vez que possibilitou 
uma formação no SUS e para o SUS, modificando a lógica do modelo privado e curativista da profissão $(28,29)$.

A implementação de políticas públicas é um processo complexo, que requer o envolvimento dos diferentes atores, entre eles, gestores, profissionais da saúde, pesquisadores, sociedade civil, entre outros e sendo assim, é fundamental que o país crie mecanismos favoráveis para sua concretização. Além disso, de acordo com o Framework “3i” as políticas públicas são baseadas no tripé (instituições, ideias e interesses), onde cada um desses componentes orientam o processo político (30).

A experiência do Brasil vem sendo motivadora e mostrou que, além de necessário é possível inserir a saúde bucal nos sistemas de saúde universais. Porém, após 14 anos e com a instabilidade política atual do país cabe a pergunta: Como será o futuro do Brasil Sorridente? Não temos a resposta, mas certamente, para que seja próspero, será necessário o compromisso dos formuladores de políticas, a continuidade do engajamento dos diferentes atores envolvidos, objetivos claros e estratégias transparentes e fundamentadas em evidências científicas para que os resultados sejam alcançados. Sobretudo, é fundamental fomentar estudos que avaliem os impactos do Brasil Sorridente em suas diferentes vertentes, ou seja, no próprio contexto político e na melhoria da saúde bucal da população, pois essa ainda é uma política nova e em construção que gradualmente vem se integrando as ações intersetoriais do SUS.

\section{REFERÊNCIAS}


1. Brasil. 1988. Constituição da República Federativa do Brasil. Brasília: Senado. Lei 8.080, de 19 de setembro de 1990. Dispõe sobre as condições para a promoção, proteção e recuperação da saúde, a organização e o funcionamento dos serviços correspondentes e dá outras providências. Diário Oficial da União, Brasília, DF, 19 set. 1990a. Seção 1.

2. Organização Pan-Americana da Saúde. Renovação da Atenção Primária em Saúde nas Américas. Documento de Posicionamento da Organização Pan - Americana da Saúde / Organização Mundial da Saúde (OPAS / OMS, 2008) [acesso em 20 ago 2018], disponível em: http://apps.who.int/medicinedocs/documents/s19055en/s19055en.pdf

3. Paim J, Travassos C, Almeida C, Bahia L, Macinko J. The Brazilian health system: history, advances, and challenges. Lancet. 2011 May 21; 377(9779): 1778-97. doi: 10.1016/S01406736(11)60054-8.

4. Brasil. Portal do Departamento de Atenção Básica. 2018. [Online]. [acesso em 20 ago 2018], disponível em:: http://dab.saude.gov.br/portaldab/pnsb.php.

5. Pucca GA Jr, Gabriel M, de Araujo ME, de Almeida FC. Ten Years of a National Oral Health Policy in Brazil: Innovation, Boldness, and Numerous Challenges. J Dent Res. 2015 Oct; 94(10): 1333-7. doi: 10.1177/0022034515599979.

6. Carrer FCA, Pucca Junior GA, Silva DP, Galante ML, Gabriel M, Macedo MCS. Observatório ibero-americano de políticas públicas em saúde bucal: construindo um bloco por mais saúde bucal. 1. ed. São Paulo: Faculdade de Odontologia da Universidade de São Paulo, 2018. v. 500. 118p. [acesso em $20 \quad$ ago 2018$], \quad$ disponível em:https://issuu.com/mainesk/docs/portugues_-_final.

7. Mendes EV. As redes de atenção à saúde. Organização Pan-Americana da Saúde, 2011. 549p.: il [acesso em 20 ago 2018], disponível em: 
http://repositorio.asces.edu.br/handle/123456789/1314

8. Chaves SCL, Almeida AMFL, Rossi TRA, Santana SF, Barros SG, Santos CML. Oral health policy in Brazil between 2003 and 2014: scenarios, proposals, actions, and outcomes. Ciênc Saúde Colet. 2017 Jun; 22(6): 1791-1803. doi: 10.1590/1413-81232017226.18782015.

9. Moysés SJ. Políticas de saúde e formação de recursos humanos em Odontologia. Rev ABENO. $2001,29(2)$.

10. Garbin CAS, Saliba NA, Santos KT. O papel das universidades na formação de profissionais na área de saúde. Rev ABENO. 2005, 3 (1).

11. Brasil. e-Gestor, informação e gestão da atenção básica. [Online]. Available at: https://egestorab.saude.gov.br/. [Acessado: 16-ago-2018].

12. Gabriel M, Cayetano MH, Chagas MM, Araújo ME, Dussault, G, Pucca Júnior GAP, Carrer FCA. Mecanismos de ingresso de cirurgiões dentistas no SUS: Uma agenda prioritária para o fortalecimento do Brasil Sorridente. Ciên Saúde Colet [Internet]. 2018. [citado 29 dez. 2018]. Disponível em: http://www.cienciaesaudecoletiva.com.br/artigos/mecanismos-de-ingressode-cirurgioes-dentistas-no-sus-uma-agenda-prioritaria-para-o-fortalecimento-do-brasilsorridente/16916?id=16916.

13. Sousa MC, Esperidião MA, Medina MG. Intersectorality in the 'Health in Schools' Program: an evaluation of the political-management process and working practices. Ciênc Saúde Colet. 2017 Jun; 22(6): 1781-90. doi: 10.1590/1413-81232017226.24262016.

14. Brasil. Programa Saúde na Escola. [Online]. [acesso em 20 ago 2018], disponível em: http://www.brasil.gov.br/editoria/saude/2017/04/programa-saude-na-escola-amplia-servicospara-estudantes.

15. Secretaria Municipal da Saude de São Paulo. Diretrizes para a Atenc! ao em Saude Bucal " - 
Crescendo e vivendo com saude bucal: [Online]. [acesso em 20 ago 2018], disponível em: http://www.prefeitura.sp.gov.br/cidade/secretarias/upload/saude/arquivos/saudebucal/Diretrizes_Saude_Bucal_2012.pdf

16. Goes PS, Figueiredo N, Neves JC, Silveira FM, Costa JF, Pucca Júnior GA, Rosales MS. [Evaluation of secondary care in oral health: a study of specialtyclinics in Brazil]. Cad Saúde Publ. 2012;28 Suppl:s81-9.

17. Brasil. Ministério da Saúde. SAGE - Sala de Apoio à Gestão Estratégica. [acesso em 20 ago 2018], disponível em: [Online]. http://sage.saude.gov.br/\#.

18. Instituto Nacional de Câncer - Estimativa 2018 - Síntese de Resultados e Comentários. 2018. [acesso em 20 ago 2018], disponível em: [Online].: http://www.inca.gov.br/estimativa/2018/sintese-de-resultados-comentarios.asp.

19. Conselho Nacional de Secretários de Saúde. Nota Técnica 26 A | 2005 Política Nacional De Atenção Oncológica. [Online]. [acesso em 20 ago 2018], disponível em: http://bvsms.saude.gov.br/bvs/publicacoes/politica_nacional_atencao_oncologica.pdf

20. IBGE. Instituto Brasileiro de Geografia e Estatística. Pesquisa nacional por amostragem de domicílios. 2010. [acesso em 20 ago 2018], disponível em: [Online] https://www.ibge.gov.br/.

21. Roncalli AG, Côrtes MI, Peres KG. [Oral health epidemiology and surveillance models in Brazil]. Cad Saúde Publ. 2012; 28 Suppl:s58-68.

22. Ministério da Saúde. 2010. Projeto SB Brasil 2010. Condições de saúde bucal da população brasileira: resultados principais. Brasília: Coordenação Nacional de Saúde Bucal, Departamento de Atenção Básica, Secretaria de Atenção à Saúde, Ministério da Saúde. [acesso em 20 ago 2018], disponível em: http://dab.saude.gov.br/portaldab/biblioteca.php?conteudo=publicacoes/pesquisa_saude_buca 
1.

23. Revista de Saúde Pública. 2013. São Paulo: Faculdade de Saúde Pública. [acesso em 20 ago 2018], disponível em: http://www.scielo.br/scielo.php?script=sci_ issuetoc\&pid=0034891020130009\&lng=pt\&nrm=iso.

24. Ministério da Saúde. Governo do estado de São Paulo. SB São Paulo 2015. Pesquisa Estadual de Saúde Bucal - Relatório Final. [Online]. [acesso em 20 ago 2018], disponível em:http://www.saude.sp.gov.br/ses/perfil/profissional-da-saude/areas-tecnicas-dasessp/saude-bucal/sb-sao-paulo-2015-pesquisa-estadual-de-saude-bucal-relatorio-final.

25. Doniec K, Dall'Alba R, King L. Brazil's health catastrophe in the making. Lancet. 2018 Jul 19. Pii; S0140-6736(18):30853-5. doi: 10.1016/S0140-6736(18)30853-5

26. da Silva JV, Machado FC, Ferreira MA. Social Inequalities and the Oral health in Brazilian Capitals. Ciênc Saúde Colet. 2015 Aug;20(8):2539-48. doi:10.1590/141381232015208.12052014.

27. de Mello AL, de Andrade SR, Moysés SJ, Erdmann AL. [Oral health care in the health network and the regionalization process]. Ciênc Saúde Colet. 2014 Jan; 19(1): 205-14. doi: 10.1590/1413-81232014191.1748.

28. Brasil. Conselho Nacional de Educação. Diretrizes Curriculares Nacionais do curso de graduação em Odontologia. Resolução CNE/ CES 3/2002. Diário Oficial da União, Brasília, DF, 04 mar. 2002.

29. Ceccin RB, Feuerwerker LM. O Quadrilátero da Formação para a Área da Saúde: Ensino, Gestão, Atenção e Controle Social. PHYSIS: Rev Saúde Coletiva. Rio de Janeiro, 2004; 14(1): $41-65$.

30. Gauvin FP. Understanding Policy Developments and Choices Through the \&quot; 3-i \&quot; 
Framework: Interests, Ideas and Institutions., Natl. Collab. Cent. Heal. Public Policy, 2014. 